\title{
Peso Bajo e insuficiente al nacer relacionado con la prevalencia de desnutrición cróni- ca en niños de tres a seis años de las instituciones educativas iniciales $N^{0} 162$ y 171 Huaycán 2012
}

\author{
Llicely Yovany BECERRIL SOPLA* \\ EAP Nutrición, Facultad de Ciencias de la Salud, Universidad Peruana Unión, Alt. Km 19; Carretera Central, Lurigancho, Lima
}

\begin{abstract}
RESUMEN
El objetivo del estudio fue determinar si existe relación entre el peso al nacer con el diagnóstico de talla para la edad en niños de tres a seis años de las Instituciones Educativas Iniciales N ${ }^{\circ} 162$ y No 171 Huaycán. El estudio fue correlacional de corte transversal; por ser un estudio poblacional, la unidad de análisis fueron todos los preescolares con una edad de 3 a 6 años, matriculado en las instituciones educativas, en total fueron 518 alumnos seleccionados por muestreo no probabilístico según criterios de inclusión y exclusión. Para la recolección de datos se usó un tallímetro y el carné de crecimiento y desarrollo del niño. Para el diagnóstico de talla para la edad, se basó en los parámetros según Waterloo y la mediana según las tablas de la OMS y para el análisis de datos se aplicó Chi-Cuadrado. Los resultados fueron que el RR obtenido de la población de estudio demuestra que a un PN bajo (1500g - 2500g) e insuficientes $(2500 \mathrm{~g}-3000 \mathrm{~g})$ aumenta el riesgo a 2,9 veces de sufrir de retardo en el crecimiento en comparación con un niño con un PN Normal. Asimismo, el RR obtenido de la población de estudio demuestra que a un PN elevado (mayor de $4000 \mathrm{~g}$ ) tiene un grado de protección moderado $(0,51)$, a sufrir de retardo en el crecimiento en comparación con un niño con un PN Normal. Por tanto, sí existe relación entre el peso al nacer y el diagnóstico de talla para la edad en la población de estudio.
\end{abstract}

Palabras clave: Peso al nacer, desnutrición, antropometría, desarrollo.

\begin{abstract}
The aim of the study was to determine the correlation between birth weight with the diagnosis of height for age in children three to six years in Initial Educational Institutions No. 162 and No. 171 Huaycán. The study was correlational cross-sectional, population-based study to be a unit of analysis were all preschool children aged 3 to 6 years, enrolled in educational institutions, a total of 518 students were selected by non-probability sampling as inclusion criteria and exclusion. To collect data and use a measuring rod license growth and development of children. For diagnosis of height for age was based on the parameters as Waterloo and the median according to WHO tables and data analysis was applied Chi-Square. The results were obtained from the RR population study shows that a low PN $(1500 \mathrm{~g}-2500 \mathrm{~g})$ and insufficient $(2500 \mathrm{~g}-3000 \mathrm{~g})$ increases to 2.9 times the risk of suffering from stunting compared to a child with BW Normal. Also, the RR obtained from the study population shows a high PN (over 4000g) has a moderate degree of protection (0.51), to suffer from stunting compared to a child with BW Normal. Thus the correlation between birth weight with the diagnosis of height for age in the study population.
\end{abstract}

Keywords: Birth weight, Malnutrition, Anthropometry, Development.

*Correspondencia: Llybs0106@gmail.com, Teléfono: 51-1989272089 


\section{INTRODUCCIÓN}

El principal activo de nuestro país es su capital humano, el cual debemos proteger desde la gestación y a lo largo de toda su existencia, con políticas económicas y sociales que garanticen su desarrollo psico-socio-espiritual logrando un desarrollo pleno de sus capacidades. Sin embargo, el principal problema de nuestra población infantil es la desnutrición, que los hace propensas a enfermedades, elevando las estadísticas de morbilidad y mortalidad, ocasionándole problemas de salud y desarrollo personal en su vida futura, lo que perjudica el desarrollo y la formación del capital humano, que en el futuro debe garantizar la continuidad de nuestro país como nación (Econ, 2008).

De acuerdo con el Censo de Población y Vivienda del año 2007 por el INEI la población infantil de 3 a 6 años en el distrito de ATE llego a 27236 habitantes.

Teniendo en cuenta que la desnutrición crónica en nuestro país aborda cifras porcentuales de $21.5 \% 3$. Asimismo, en distrito de Vitarte alcanza a más de 13390.2 mil niños, que tendrán un menor desarrollo físico y verán seriamente afectadas sus capacidades cognoscitivas y estarán más expuestos a enfermedades, lo que continuará afectando el capital humano de nuestro país, puesto que éste no es un problema nuevo (Arlette, 2009).

Por otro lado, los estudios demuestran que el peso al nacer es una de las variables reconocidas entre las de mayor importancia, por su asociación al desarrollo y crecimiento durante la infancia y en la vida adulta como pre disponente de enfermedades crónicas. Así por ejemplo, un peso al nacer bajo y un recién nacido macroscópico, aumentan las posibilidades de desarrollar diversas enfermedades crónicas en la vida adulta. Siendo así que un recién nacido con bajo peso, aumenta el riesgo de padecer enfermedades y de mantener un estado nutricional inadecuado durante su crecimiento, también que pueden padecer de trastornos de tipo neurológico incluyendo el déficit intelectual, en comparación con niños nacidos con un peso adecuado.

Además los niños con problemas en el crecimiento (talla) alcanza a más de 5855.74 mil niños, que tendrán un menor desarrollo físico y se verán seriamente afectadas sus capacidades físicas, cognoscitivas y sociales, convirtiéndoles en niños más propensos a enfermedades, con un menor desarrollo social, lo que continuará afectando el capital humano de nuestro país (INEI, 2007).

El objetivo del estudio fue determinar si existe relación entre el peso al nacer con el diagnóstico de talla para la edad en niños de tres a seis años de las Instituciones Educativas Iniciales № 162 y Nº 171 Huaycán.

\section{MATERIALES Y MÉTODOS}

El estudio de diseño no experimental, tipo correlacional y transversal. El trabajo se desarrolló en las instituciones iniciales I.E.I N 162 y N 171 , ubicadas en la zona A y zona $\mathrm{G}$ respectivamente en la Comunidad Urbana Autogestionaria de Huaycán que se encuentra ubicada en el distrito de Ate, al este de la provincia de Lima, a la altura del kilómetro 16,5 de la Carretera Central. El distrito de Huaycán es el hogar de miles de pobladores inmigrantes de diferentes partes del Perú quienes habiéndose agrupado en 23 organizaciones llegaron a Huaycán en busca de mejorar su nivel de vida y oportunidades de trabajo en la ciudad de Lima.

\section{Descripción de los centros educativos}

\section{Institución Educativa Inicial No 162}

El centro educativo cuenta con una infraestructura de ocho ambientes para sala de clases implementados adecuadamente los cuales se identifican con nombre de colores (Amarillo, Melón, Celeste, Lila, Rojo, Rosado, Anaranjado y Verde); una sala para asistencia (psicológica, nutricional, psicomotora), un área de esparcimiento (para la realización de reuniones, formación y deporte), una ambiente de cocina (donde se solía preparar la alimentación otorgada por el PRONA) y una oficina para la dirección. La Institución Educativa Inicial $\mathrm{N}^{\circ} 162$ tiene 25 años de funcionamiento, brindando a diversas generaciones conocimientos y hábitos que quedaran arraigados a lo largo de toda su vida. El centro cuenta con 14 trabajadores conformados, por la directora, las profesoras del aula y sus auxiliares.

\section{Institución Educativa Inicial No 171}

El centro educativo cuenta con una infraestructura de nueve ambientes para sala de clases implementados adecuadamente las cuales se identifican con nombre de valores (Responsabilidad, Honestidad, Justicia, Veracidad, Solidaridad, Amistad, Lealtad, Humildad y Tolerancia; un área de esparcimiento (para la realización de reuniones, formación y deporte), una ambiente de cocina, un aula de cómputo y una oficina para la dirección. La Institución Educativa Inicial $\mathrm{N}^{\circ} 171$ tiene 22 años de funcionamiento, brindando a diversas generaciones conocimientos y hábitos que quedarán arraigados a lo largo de toda su vida. El centro cuenta con 19 trabajadores conformados, por la directora, las profesoras del aula y sus auxiliares.

La población de este estudio estuvo constituida por 542 de la niños y niñas de tres a seis años matriculados en la I.E.I. Nº 162 y la I.E.I. $N^{\circ} 171$ de Huaycán. Por ser un estudio poblacional la unidad de análisis fueron todos los preescolares con una edad de 3 a 6 años, 
matriculado en la institución educativa inicial $N^{\circ} 162$ y № 171 de Huaycán. Para la selección de la unidad muestral se utilizó el muestreo no probabilístico por conveniencia, debido a que luego de la evaluación antropométrica se incluyó dentro de este estudio a todo preescolar, que tengan como diagnóstico antropométrico: “desnutrición crónica”, en cualquier grado, niños que asistan de forma regular a clases, con edades de tres a seis años.

Quedando una muestra de 518 niños de los cuales el $50,2 \%$ fueron varones y el $49,8 \%$ mujeres. Las edades fueron comprendidos de 3 años, (36meses) a menos de 6 años (72 meses).

\section{RESULTADOS Y DISCUSIÓN}

\section{Tabla 1}

Tipo de retardo de crecimiento en los niños y niñas de las I.E.I $N^{o} 162$ y 171

\begin{tabular}{ccc}
\hline Tipo de retardo & $\mathrm{N}$ & $\%$ \\
\hline Retardo en el crecimiento moderado & 8 & 1,5 \\
Retardo en el crecimiento leve & 142 & 27,4 \\
Normal & 368 & 71,0 \\
Total & 518 & 100,0 \\
\hline
\end{tabular}

En la tabla 1, que el 1,5\% de los niños tiene retardo en el crecimiento moderado y el 27,4\% retardo en el crecimiento leve.

Tabla 2

Peso al nacer (PN) en los niños y niñas de las I.E.I $N^{o} 162$ y 171

\begin{tabular}{ccc}
\hline Peso al nacer $(\mathrm{PN})$ & $\mathrm{N}$ & $\%$ \\
\hline PN bajo & 22 & 4,2 \\
PN insuficiente & 86 & 16,6 \\
PN normal & 365 & 70,5 \\
PN Elevado & 45 & 8,7 \\
Total & 518 & 100,0 \\
\hline
\end{tabular}

Como muestra la tabla 2, el 20.8\% de los niños evaluados presentó un peso al nacer por debajo de lo normal, comprendido por un 4,2\% de PN bajo (1500g-2500g) y $16.6 \%$ PN insuficiente $(2500 \mathrm{~g}-3000 \mathrm{~g})$ y un $70.5 \%$ de los niños evaluados presentó un peso al nacer normal.

Tabla 3

Riesgo relativo RR de las variables peso bajo e insuficiente al nacer con la prevalencia de desnutrición crónica

\begin{tabular}{cccc}
\hline & Casos & Controles & \\
\hline Expuestos & $\mathrm{a}$ & $\mathrm{b}$ & 108 \\
PN Bajo e insuficiente & 67 & 41 & 365 \\
No expuestos & $\mathrm{c}$ & $\mathrm{d}$ & \\
PN Normal & 78 & 287 & \\
$\mathrm{RR}=$ & $\mathrm{A} /(\mathrm{A}+\mathrm{B})$ & & \\
\cline { 2 - 2 } $\mathrm{C} /(\mathrm{C}+\mathrm{D})$ & & \\
\cline { 2 - 3 } & 0.62037037 & \\
\end{tabular}


La tabla 3 muestra que los niños que nacen con peso bajo o insuficiente (1500 a 2500 y de 2500 a $3000 \mathrm{~g}$ respectivamente), tienen 2.9 veces más riesgo de presentar retardo en el crecimiento que aquellos niños que nacen con un peso normal.

\section{Tabla 4}

Riesgo relativo $R R$ de retardo en el crecimiento, para niños con antecedentes de elevado peso al nacer (más de 4000g).

\begin{tabular}{|c|c|c|c|}
\hline & Casos & Controles & \\
\hline Expuestos & $\mathrm{a}$ & $\mathrm{b}$ & \\
\hline PN Elevado & 5 & 40 & 45 \\
\hline No expuestos & c & d & \\
\hline PN Normal & 78 & 287 & 365 \\
\hline \multirow[t]{2}{*}{$\mathrm{RR}=$} & $\mathrm{A} /(\mathrm{A}+\mathrm{B})$ & & \\
\hline & $\mathrm{C} /(\mathrm{C}+\mathrm{D})$ & & \\
\hline \multirow[t]{2}{*}{$\mathrm{RR}=$} & 0.111111111 & & \\
\hline & 0.21369863 & & \\
\hline $\mathrm{RR}=$ & 0.51994302 & & \\
\hline
\end{tabular}

La tabla 4, muestra que los niños que nacen con un peso elevado mayor de $4000 \mathrm{~g}$, tienen 0,51 veces menos de riesgo de presentar retardo en el crecimiento. En otras palabras el nacer con un peso superior a 4000g resulta ser un factor de protección contra el retardo en el crecimiento.

Tabla 5

Relación entre el peso al nacer con el diagnostico de talla para la edad, en niños de tres a seis años

\begin{tabular}{cccc}
\hline & Valor & gl & Sig. asintótica (bilateral) \\
\hline Chi-cuadrado de Pearson & $55,809(\mathrm{a})$ & 6 &, 000 \\
Razón de verosimilitudes & 53,693 & 6 &, 000 \\
Asociación lineal por lineal & 43,817 & 1 &, 000 \\
\hline
\end{tabular}

La tabla 5 confirma la relación significativa con un $\operatorname{sig}=.000$, que indica que sí existe relación entre el peso al nacer con el diagnostico de talla para la edad, en niños de tres a seis años de edad. 


\section{REFERENCIAS BIBLIOGRÁFICAS}

1. Arlette B. (2009). Desnutrición crónica infantil en el Perú. Centro de Investigación de la Universidad del Pacífico. Recuperado de: http://www.slideshare.net/cic_ucab/desnutricion-crnica-infantil-en-elper-un-problema-persistente.

2. Econ. J. \& Sifuentes Z. (2008). Desnutrición infantil en Perú. 2008. Disponible en http://www. cel.org.pe/articulos/desnutricion\%20INFANTIL\%20EN\%20EL \%20PERU.pdf.

3. INEI (2007). Factores asociados a la desnutrición crónica infantil en el Perú, 1996 - 2007. Recuperado de http://www.inei.gob.pe/biblioineipub/ bancopub/ Est/ Lib0893/Libro.pdf.

4. INEI (2007). Censo de Población y Vivienda. Recuperado de http:// iinei.inei.gob.pe/iinei/RedatamCpv2007.asp?id=ResultadosCensales?ori=C.

5. INEI (2007).Tendencia de riesgo de nacer con muy bajo peso en el Perú y factores condicionales. Recuperado de: http://www.inei.gob.pe/biblioineipub/ bancopub/Est/Lib0566/Libro.pdf. 